\title{
Inhibition of human lung cancer proliferation through targeting stromal fibroblasts by dihydromyricetin
}

\author{
KAI-JIE FAN ${ }^{*}$, BO YANG ${ }^{*}$, YANG LIU, XIAO-DONG TIAN and BO WANG \\ Department of Thoracic Surgery, PLA General Hospital, Beijing 100853, P.R. China
}

Received May 23, 2016; Accepted August 10, 2017

DOI: $10.3892 / \mathrm{mmr} .2017 .7802$

\begin{abstract}
In the present study, the effects of dihydromyricetin on the proliferative potential of fibroblasts and lung carcinoma cells were investigated. Markedly higher expression levels of smooth muscle actin and platelet derived growth factors (PDGFs) were observed in the fibroblasts using reverse transcription-polymerase chain reaction analysis. The expression levels of PDGF-A and PDGF-B were also higher in the lung cancer cells. Western blot analysis revealed higher expression levels of the receptor for platelet-derived growth factor (PDGFR $\beta$ ) in the lysates from fibroblasts obtained from normal tissues and carcinoma tissues. Treatment of the fibroblasts with dihydromyricetin inhibited the expression of PDGFR $\beta$ when treated with a $10 \mu \mathrm{M}$ concentration for $48 \mathrm{~h}$. Treatment of the fibroblasts with a $10 \mu \mathrm{M}$ concentration of dihydromyricetin for $48 \mathrm{~h}$ led to complete inhibition of the activation of extracellular signal-regulated kinase (Erk)1/2 and Akt. The results of an MTT assay showed that treatment of the fibroblasts with dihydromyricetin significantly reduced the PDGF-mediated increase in the rate of proliferation. The rate of proliferation of the A549 lung cancer cells cultured with fibroblasts was markedly increased, compared with that of the A549 cells cultured alone. However, dihydromyricetin significantly $(\mathrm{P}<0.05)$ inhibited the proliferation rate of the A549 cells cultured with fibroblasts, compared with the untreated cultures. The proliferation rates of the A549 cancer cells, A549 cells cultured with fibroblasts, and A549 cells cultured with fibroblasts and treated with dihydromyricetin were found to be were $78.45,98.45$ and $21.37 \%$, respectively. Dihydromyricetin inhibited the proliferative potential of fibroblasts in the lung cancer cells through targeting the activation of Erk1/2 and Akt. Therefore, there is scope for
\end{abstract}

Correspondence to: Dr Yang Liu, Department of Thoracic Surgery, PLA General Hospital, 28 Fuxing Road, Beijing 100853, P.R. China

E-mail: yangliu231@hotmail.com

*Contributed equally

Key words: stroma, fibroblasts, proliferative, platelet, receptor, lung cancer dihydromyricetin to be evaluated further for the treatment of lung cancer.

\section{Introduction}

Lung cancer is a commonly diagnosed cancer in developed countries and has poor rate of prognosis (1). The treatments currently available for lung cancer include surgical extraction at an early stage and the use of the chemotherapeutic agents (2-4). However, despite these strategies, tumor recurrence is reported in the majority of patients (2-4). Therefore, the development of novel efficient treatment strategies for lung cancer is warranted. A tumor mass consists of a combination of carcinoma cells, fibroblasts and inflammatory cells (5). Stromal fibroblasts possess markedly higher levels of a protein known as $\alpha$-smooth muscle actin ( $\alpha$-SMA) (6,7). These stromal fibroblasts secrete various factors, which enhance the rate of carcinoma cell proliferation, and increase the invasive and migration potential of cancer cells (8-11). Studies have revealed that stromal formation in various types of malignant carcinoma is facilitated by platelet-derived growth factor (PDGF) (12-14), and there are interactions between receptors for tyrosine kinase and PDGF (15).

Natural products offer promise in the treatment of various types of diseases without producing harmful side effects due to their modification in the living systems over millions of years. Dihydromyricetin (Fig. 1), a member of the flavonoid family, was obtained following the phytochemical investigation of Ampelopsis grossedentata (16). The biological evaluation of dihydromyricetin has shown its promising potential as an anti-oxidant, antithrombolytic and anti-inflammatory agent (16-18). Dihydromyricetin has been shown to inhibit the peroxidation of membrane lipids in various types of cell and also act as hepatoprotective agent $(19,20)$. The present study was performed to investigate the effect of dihydromyricetin on the growth of fibroblasts in lung carcinoma. The results demonstrated that dihydromyricetin inhibited the proliferation of fibroblasts through the inhibition of extracellular signalregulated kinase (Erk)1/2 and Akt.

\section{Materials and methods}

Reagents and drugs. Dihydromyricetin and dimethyl sulfoxide (DMSO) were purchased from Sigma-Aldrich; Merck Millipore (Darmstadt, Germany). PDGF-BB was obtained 
from Gibco; Thermo Fisher Scientific, Inc. (Waltham, MA USA).

Cell lines and culture. The A549 lung carcinoma cell line was obtained from the China Center for Type Culture Collection (Wuhan, China). The A549 cells were cultured in Dulbecco's modified Eagle's medium (Gibco; Thermo Fisher Scientific, Inc.) containing $10 \%$ fetal bovine serum (FBS; Gibco; Thermo Fisher Scientific, Inc.). The medium was supplemented with antibiotics streptomycin $(50 \mathrm{mg} / \mathrm{ml})$ and penicillin $(50 \mathrm{IU} / \mathrm{ml})$, and glutamine $(2 \mathrm{mM})$. The cells were cultured in a humidified atmosphere of $5 \% \mathrm{CO}_{2}$ and $95 \%$ air at $37^{\circ} \mathrm{C}$.

Fibroblast culture. The lung carcinoma tissue samples were obtained immediately following collection and were stored under a liquid nitrogen atmosphere. Written consent was obtained from the patients ( 4 patients, 3 males and 1 female in the age group of 45-60 years with lung cancer admitted in the PLA General Hospital, Beijing China on 23-04-2016) confirming that they were aware of the type of investigation being performed. The study was approved by the local ethics committee of PLA General Hospital (Beijing, China). The thin lung tissue samples ( $30 \mu \mathrm{M}^{3}$ thickness) were subjected to incubation for $1 \mathrm{~h}$ in DMEM/F-12 supplemented with dispase $(2,500 \mathrm{U} / \mathrm{ml})$ and collagenase Type IV (450 U/ml). Following incubation, the primary fibroblasts were then cultured in DMEM/F-12 supplemented with penicillin $(50,000 \mathrm{U} / \mathrm{ml})$ and streptomycin $(50,000 \mu \mathrm{g} / \mathrm{ml})$. The fibroblasts were cultured overnight in a humidified atmosphere of $5 \% \mathrm{CO}_{2}$ and $95 \%$ air at $37^{\circ} \mathrm{C}$. The fibroblasts obtained from the normal and carcinoma tissues were designated as FKs and CKs, respectively.

Immunohistochemistry. The lung cancer tissue samples were washed with PBS and then embedded in paraffin. The tissues were sectioned into $2-\mu \mathrm{m}$-thin sections, boiled in xylene and rehydrated in gradient ethanol. The activity of endogenous peroxidase in the sections was quenched by treatment with $0.3 \% \mathrm{H}_{2} \mathrm{O}_{2}$. The sections were then incubated with primary antibodies against $\alpha$-SMA (cat. no. A03744; dilution 1:200; Boster Biological Technology, Wuhan, China) for $2 \mathrm{~h}$ at room temperature. Visualization of the samples was performed using a Histofine Simple Stain MAX-PO (Multi) and DAB substrate kit (Nichirei Biosciences, Inc., Tokyo, Japan). The counterstaining was performed using hematoxylin at $37^{\circ} \mathrm{C}$ for $45 \mathrm{~min}$ and images were captured with a light microscope (Nikon Eclipse E800; Berlin, Germany) equipped with a Spot RT digital camera (Diagnostic Instruments Inc, Sterling Heights, CA, USA).

Reverse transcription-polymerase chain reaction (RT-PCR) analysis. From the fibroblasts and the A549 cells treated with dihydromyricetin for $48 \mathrm{~h}$, total RNA was isolated using TRIzol reagent (Beyotime Institute of Biotechnology, Shanghai, China). The first-strand cDNA synthesis was performed using reverse transcriptase (Beyotime Institute of Biotechnology) with $2 \mu \mathrm{g}$ samples of RNA. The Premix Ex Taq kit (Takara Biotechnology Co., Ltd., Dalian, China) was used for performing the RT-PCR analysis. Transcript amplification was carried out by PCR as follows: $2 \mu \mathrm{l}$ template complimentary DNA (cDNA), $0.2 \mathrm{mM}$ dNTPs, $1.25 \mathrm{U} / \mu \mathrm{l}$ Go

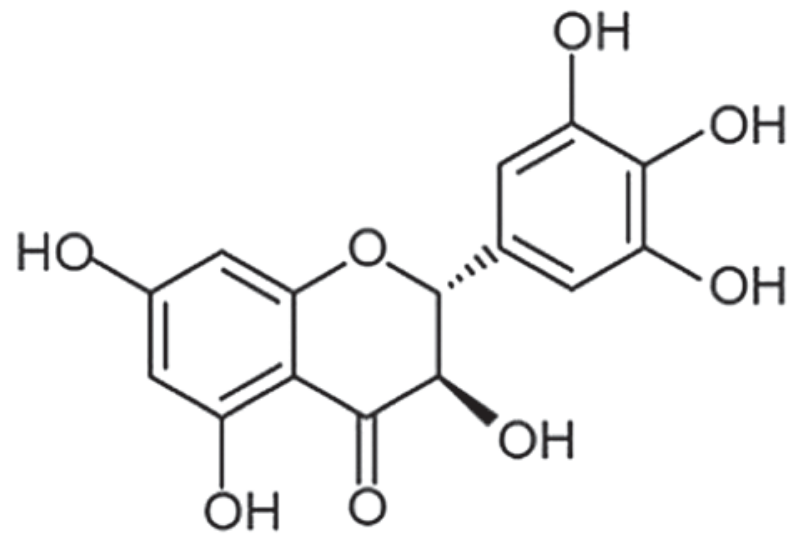

Figure 1. Chemical structure of dihydromyricetin.

Taq polymerase (Promega, Corporation, Madison, WI, USA) and various primer sets $(0.5 \mu \mathrm{M}$ each $)$ in $50 \mu \mathrm{l}$ water. The denaturation was performed for $5 \mathrm{~min}$ prior to 40 thermal cycles of $95^{\circ} \mathrm{C}$ for $1 \mathrm{~min}, 52^{\circ} \mathrm{C}$ for $25 \mathrm{sec}$ and $75^{\circ} \mathrm{C}$ for $1 \mathrm{~min}$, with a final extension step at $75^{\circ} \mathrm{C}$ for $10 \mathrm{~min}$. Following amplification, the PCR products were subjected to electrophoresis on $1 \%$ agarose gels at $80 \mathrm{~V}$ for $45 \mathrm{~min}$ and were subsequently visualized using ethidium bromide staining. The primer sequences are as follows: $\alpha$-SMA forward, 5'-CCAACCGGGAGAAAATGA CTCAAATT-3' and reverse, 5'-AGGCAACTCGTAACTCTT CTCAAGG-3'; FAP forward, 5'-CCAGCAATGATAGCC TCAA-3' and reverse, 5'-TAACACACTTCTTGCTTGGA-3'; PDGFR $\alpha$ forward, 5'-GAAGCTGTCAACCTGCATGA-3' and reverse, 5'-CTTCCTTAGCACGGATCAGC-3'; PDGFR $\beta$ forward, 5'-GTGAACGCAGTGCAGACTGT-3' and reverse, 5'-AGGTGTAGGTCCCCGAGTCT-3'; GAPDH Forward, 5'-TGAAGGTCGGAGTCAACGGATTTGGT-3' and reverse, 5'-CATGTGGGCCATGAGGTCCACCAC-3'.

Western blot analysis. The expression levels of factors, including PDGFR $\beta$, p-PDGFR $\beta$, Akt, p-Akt Erk1/2 and p-Erk1/2, were analyzed in the fibroblasts following treatment with dihydromyricetin. The FKs and CKs were treated with lysis buffer under ice-cold conditions for $45 \mathrm{~min}$. The lysates were then centrifuged at $12,000 \times \mathrm{g}$ for $20 \mathrm{~min}$ at $4^{\circ} \mathrm{C}$ and the concentration of proteins in the supernatants was determined using a bicinchoninic assay. Equal quantities of the cell lysates $(40 \mu \mathrm{g}$ protein) were subjected to separation via SDS-PAGE on $10 \%$ a gel and subsequently transferred onto polyvinylidene difluoride membranes. Blocking of the membranes was performed by incubation with $5 \%$ non-fat milk at $37^{\circ} \mathrm{C}$ for $1 \mathrm{~h}$. The membranes were then incubated with PDGF receptor $\beta$ (PDGFR $\beta$; cat. no. P3082; dilution 1:1,000), Erk1/2 (cat. no.62206; dilution 1:1,000), phosphorylated (p)-Akt (cat. no. KHO0201; dilution 1:1,000; all the three from Thermo Fisher Scientific Inc.; MK3), Akt (cat. no. 200-401-N98; dilution 1:1,000; Rockland, Gilbertsville, PA, USA), p-PDGFR $\beta$ (ab16868; dilution 1:1,000; Abcam) and p-Erk1/2 (FCMAB100P; dilution 1:1,000; EMD Millipore (Darmstadt, Germany) primary antibodies (at 1:1000 dilution) overnight at $4^{\circ} \mathrm{C}$ and washed with TBST for 5 min. $\beta$-actin (1:500, sc-47778; Santa Cruz Biotechnology Inc., Dallas, TX, USA) was used as an internal loading control. The membranes were then incubated with horseradish peroxidase-conjugated 

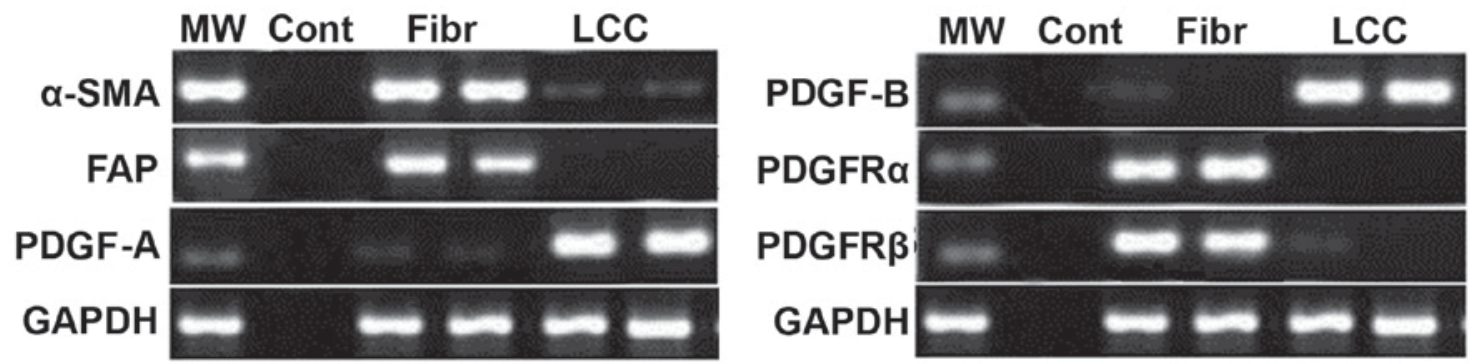

Figure 2. Expression levels of $\alpha$-SMA, PDGF-A, PDGF-B and its PDGFR in fibroblasts and lung cancer cells. The expression of the negative control, fibroblasts and lung cancer cells were analyzed using reverse transcription-polymerase chain reaction analysis to determine the expression levels of these factors. $\alpha$-SMA, $\alpha$-smooth muscle actin; FAP, fibroblast activation protein; PDGF, platelet-derived growth factor; PDGFR, PDGF receptor; MW, marker; Con, negative control; Fibr, fibroblasts; LCC, lung cancer cells.
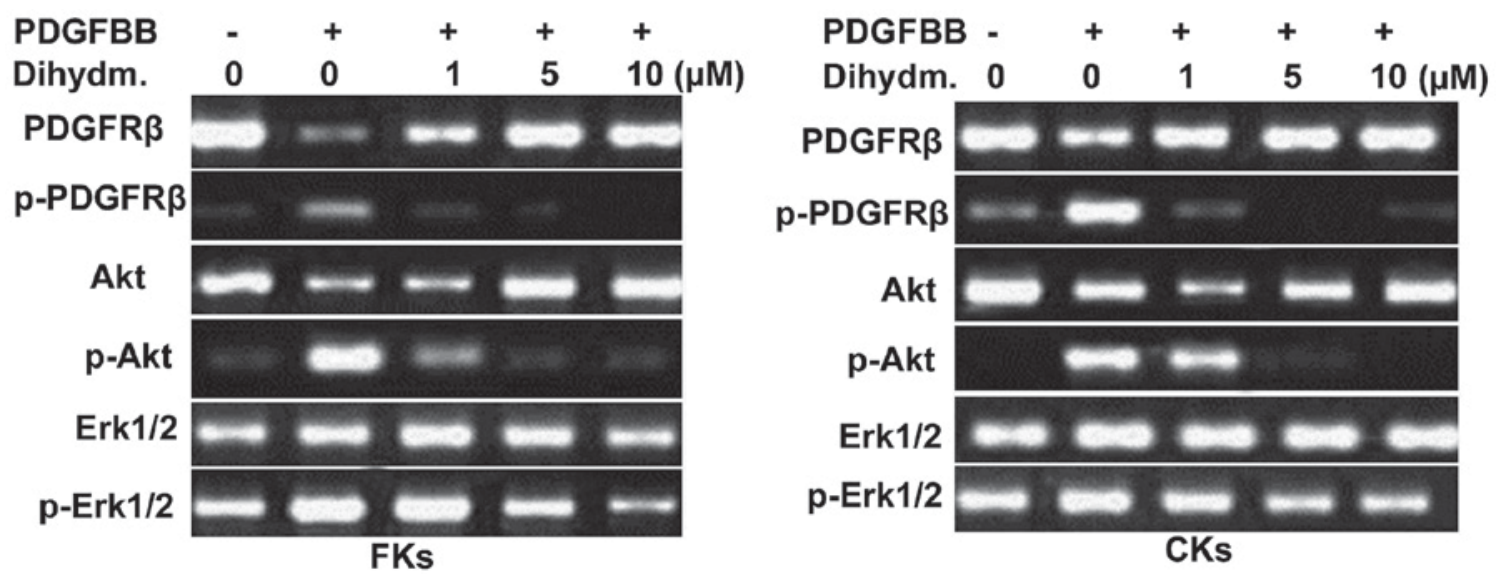

Figure 3. Dihydromyricetin alters the expression levels of PDGFR $\beta$ and the activation of Akt and Erk1/2 in fibroblasts. The fibroblasts were treated with various concentrations of dihydromyricetin or with dimethyl sulfoxide alone (control) and were then subjected to reverse transcription-polymerase chain reaction analysis. PDGF, platelet-derived growth factor; PDGFR, PDGF receptor; Dihydm, dihydromyricetin; Erk, extracellular signal-regulated kinase; p-, phosphorylated; FKs, fibroblasts from normal tissue; CKs, fibroblasts from cancerous tissue.

secondary antibodies (cat. no. MPX-2402; 1:5,000 dilution in TBST containing $1 \%$ bovine serum albumin; Santa Cruz Biotechnology, Inc.) at room temperature for $1 \mathrm{~h}$. Analysis of the bands was performed using an enhanced chemiluminescence blotting detection system (FluorChem E; Proteinsimple, Santa Clara, CA, USA).

Cell proliferation assay. The effects of dihydromyricetin treatment on the rates of lung cancer cell and fibroblast proliferation were analyzed using an MTT assay. The cells were distributed at a density of $2 \times 10^{6}$ cells per well in 96-well culture plates and incubated for $24 \mathrm{~h}$ at $37^{\circ} \mathrm{C}$. Following incubation, the medium was replaced with medium containing various concentrations $(1,5,10 \mu \mathrm{M})$ of dihydromyricetin. The cells in the control wells were treated with DMSO alone. After incubation for $48 \mathrm{~h}$ at $37^{\circ} \mathrm{C}$, MTT solution $(5 \mathrm{mg} / \mathrm{ml})$ was added into each well of the plate and the plates were incubated at a temperature of $37^{\circ} \mathrm{C}$ for $4 \mathrm{~h}$. The culture medium was removed carefully and DMSO was added to solubilize the formazan crystals. The absorbance measurement for each well was performed at $570 \mathrm{~nm}$ using a microplate reader (PerkinElmer, Inc., Waltham, MA, USA). All experiments were performed in triplicate.

Statistical analysis. All data are presented as the mean \pm standard deviation of experiments performed in triplicate.
Comparisons among multiple groups were performed using a one-way analysis of variance. Statistical analysis was performed using SPSS 180 statistical software (SPSS, Inc., Chicago, IL, USA). In all cases, comparisons were made relative to untreated controls. $\mathrm{P}<0.05$ was considered to represent a statistically significant difference.

\section{Results}

Levels of PDGFR and PDGF in lung carcinoma cells and fibroblasts. The results from the RT-PCR analysis showed markedly higher expression of $\alpha$-SMA and fibroblast activation protein in the fibroblasts (Fig. 2). The expression of PDGF-A and PDGF-B were markedly lower in the fibroblasts compared to lung carcinoma cells. In the lung cancer cells, the expression levels of PDGFR $\alpha$ and PDGFR $\beta$ were markedly lower in comparison to the levels in the fibroblasts (Fig. 2).

Effect of dihydromyricetin on the PDGFR activity of tyrosine kinase in fibroblasts. The results from western blot analysis of the lysates from the FK and CK fibroblasts showed markedly higher activity of PDGFR $\beta$ (Fig. 3). Treatment of the fibroblasts with different concentrations of dihydromyricetin caused suppression in the activity of PDGFRß in a dose-dependent manner. The inhibited activation of PDGFR $\beta$ was maximal 


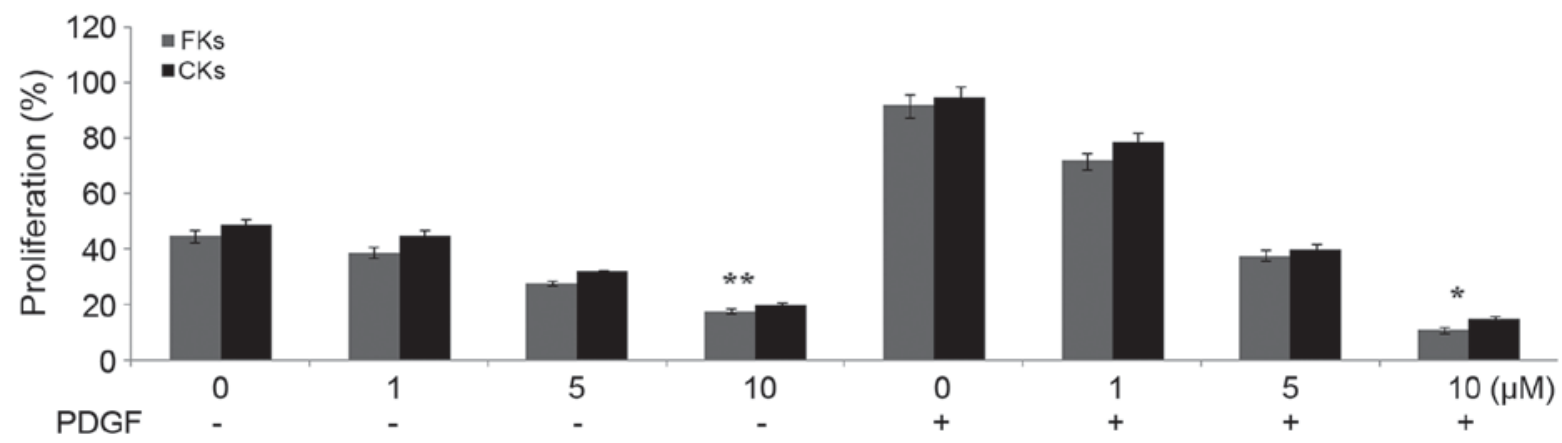

Figure 4. Dihydromyricetin inhibits the PDGF-induced increase in the proliferation rate of fibroblasts in a dose-dependent manner. The fibroblasts were incubated with dimethyl sulfoxide, PDGF and various concentrations of dihydromyricetin for $48 \mathrm{~h}$ and then analyzed using an MTT assay. The experiments were performed in triplicate and the values are presented as the mean. ${ }^{*} \mathrm{P}<0.05$ and ${ }^{* *} \mathrm{P}<0.01$ compared to control cells. PDGF, platelet-derived growth factor; FKs, fibroblasts from normal tissues; CKs, fibroblasts from cancerous tissues.

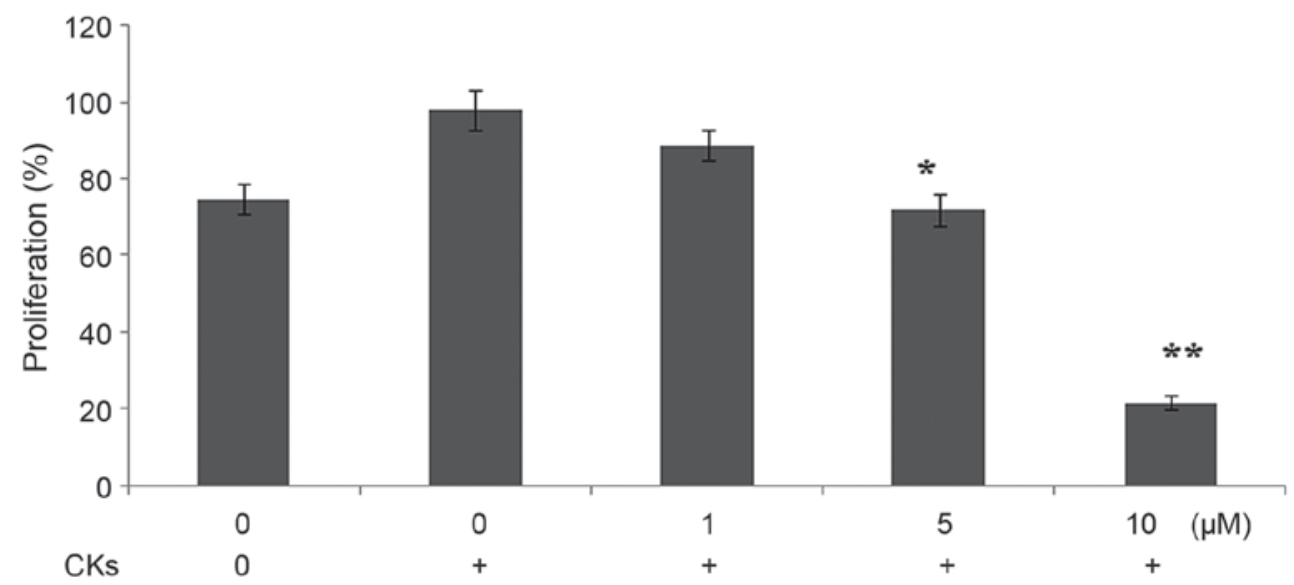

Figure 5. Dihydromyricetin inhibits the fibroblast-induced increase in the proliferation potential of lung cancer cells. The cells were incubated with dimethyl sulfoxide (control), fibroblasts or dihydromyricetin and fibroblasts and then analyzed using MTT assay. The absorbance measurements were performed in triplicate and the values are presented as the mean. ${ }^{*} \mathrm{P}<0.05$ and ${ }^{* *} \mathrm{P}<0.01$ compared to the control lung cancer cells. CKs, fibroblasts from cancerous tissues.

following treatment at a dihydromyricetin concentration of $10 \mu \mathrm{M}$ for $48 \mathrm{~h}$. However, dihydromyricetin caused no alteration in the expression of the protein, PDGFR $\beta$ in the fibroblasts (Fig. 3).

Effect of dihydromyricetin on the activation of phosphoinositide 3-kinase-Akt and Ras-mitogen-activated protein kinase (MAPK) Erk1/2. Incubation of the FK and CK fibroblasts with PDGF-BB caused a significant increase in the activation of Erk1/2 and Akt. Dihydromyricetin treatment for $48 \mathrm{~h}$ led concentration-dependent inhibition of the activation of Erk1/2 and Akt in the fibroblasts (Fig. 3). The activation of Erk1/2 and Akt in the fibroblasts was completely inhibited following $48 \mathrm{~h}$ of treatment with a $10 \mu \mathrm{M}$ concentration of dihydromyricetin.

Effect of dihydromyricetin on the proliferation of fibroblasts induced by PDGF. The results of the MTT assay showed a significant increase in the proliferation rates of the FK and CK fibroblasts following incubation with PDGF. Treatment of the fibroblasts with dihydromyricetin reduced the PDGF-mediated increase in the rates of proliferation (Fig. 4). The fibroblasts were treated with various concentrations of dihydromyricetin and the maximum inhibition of proliferation was observed with a concentration of $10 \mu \mathrm{M}$.
Effect of dihydromyricetin on the proliferation rate of lung cancer cells cultured with fibroblasts. The rate of proliferation of the A549 lung cancer cells cultured with CK fibroblasts was higher, compared with that in A549 cells cultured alone. However, dihydromyricetin significantly $(\mathrm{P}<0.05)$ inhibited the rate of proliferation of A549 cells cultured with CKs, compared with the untreated cultures (Fig. 5). The proliferation rates of the A549 cancer cells, A549 cells cultured with CK fibroblasts and A549 cells cultured with CK fibroblasts and treated with dihydromyricetin were $78.45,98.45$ and $21.37 \%$, respectively.

\section{Discussion}

The aim of the present study was to investigate the effect of dihydromyricetin on the proliferation of fibroblasts in lung carcinoma. The study demonstrated the inhibition of fibroblast proliferation by dihydromyricetin via targeting the activation of Erk1/2 and Akt.

The tumor stroma exhibits a promoting effect on the development of carcinoma, in which fibroblasts are the major contributing factor (8-11). It has been suggested that fibroblasts constitute a vital target for the treatment of tumors (21-24). The present study revealed that incubation of lung 
cancer cells with fibroblasts markedly increased the rate of proliferation, compared with cancer cells cultured alone. However, the fibroblast-induced proliferation of lung cancer cells was significantly inhibited following treatment with dihydromyricetin, in a concentration-dependent manner. There are reports that carcinoma cells produce PDGFs, which enhance the proliferation rate of fibroblasts in various tumor xenograft carcinoma models, including breast cancer, lung cancer, melanoma and colorectal cancer $(12,13,25,26)$. The results of the present study demonstrated markedly higher expression levels of $\alpha$-SMA and fibroblast activation protein in the fibroblasts. The expression of PDGF-A was also markedly higher in the fibroblasts. The activities of PDGFR $\beta$ in the lysates from FK and CK fibroblasts were also markedly higher, as revealed using western blot analysis. Treatment of the fibroblasts with different concentrations of dihydromyricetin caused suppression of PDGFR $\beta$ in a dose-dependent manner. However, dihydromyricetin did not alter the protein expression of PDGFR $\beta$ in fibroblasts.

The incubation of FK and CK fibroblasts with PDGF-BB caused a significant increase in the activation of Erk1/2 and Akt. Dihydromyricetin treatment for $48 \mathrm{~h}$ led to a concentration-dependent inhibition of the activation of Erk1/2 and Akt in the fibroblasts. The MTT assay showed a significant increase in the proliferation rates of the FK and CK fibroblasts following incubation with PDGF. Treatment of the fibroblasts with dihydromyricetin reduced the PDGF-mediated increase in the rate of proliferation. The fibroblasts were treated with various concentrations of dihydromyricetin and the maximum inhibition of proliferation was observed at a concentration of $10 \mu \mathrm{M}$.

In conclusion, dihydromyricetin inhibited the proliferative potential of fibroblasts on lung cancer cells through targeting the activation of Erk1/2 and Akt. Therefore, dihydromyricetin offers potential for further evaluation for the treatment of lung cancer.

\section{References}

1. Jemal A, Siegel R, Ward E, Hao Y, Xu J, Murray T and Thun MJ: Cancer statistics. CA Cancer J Clin 58: 71-96, 2008.

2. Asamura H, Goya T, Koshiishi Y, Sohara Y, Eguchi K, Mori M, Nakanishi Y, Tsuchiya R, Shimokata K, Inoue H, et al: A Japanese lung cancer registry study: Prognosis of 13,010 resected lung cancers. J Thorac Oncol 3: 46-52, 2008.

3. Mountain CF: Revisions in the international system for staging lung cancer. Chest 111: 1710-1717, 1997.

4. Tsuboi M, Ohira T, Saji H, Miyajima K, Kajiwara N, Uchida O, Usuda J and Kato H: The present status of postoperative adjuvant chemotherapy for completely resected non-small cell lung cancer. Ann Thorac Cardiovasc Surg 13: 73-77, 2007.

5. Tremblay G: Stromal aspects of breast carcinoma. Exp Mol Pathol 31: 248-260, 1979.

6. Gabbiani G, Ryan GB and Majne G: Presence of modified fibroblasts in granulation tissue and their possible role in wound contraction. Experientia 27: 549-550, 1971.

7. Rønnov-Jessen L, Petersen OW, Koteliansky VE and Bissell MJ: The origin of the myofibroblasts in breast cancer. Recapitulation of tumor environment in culture unravels diversity and implicates converted fibroblasts and recruited smooth muscle cells. J Clin Invest 95: 859-873, 1995.
8. Olumi AF, Grossfeld GD, Hayward SW, Carroll PR, Tlsty TD and Cunha GR: Carcinoma-associated fibroblasts direct tumor progression of initiated human prostatic epithelium. Cancer Res 59: 5002-5011, 1999

9. Okusa Y, Ichikura T and Mochizuki H: Prognostic impact of stromal cell-derived urokinase-type plasminogen activator in gastric carcinoma. Cancer 85: 1033-1038, 1999.

10. Orimo A, GuptaPB,Sgroi DC,Arenzana-Seisdedos F, Delaunay T, Naeem R, Carey VJ, Richardson AL and Weinberg RA: Stromal fibroblasts present in invasive human breast carcinomas promote tumor growth and angiogenesis through elevated SDF-1/CXCL12 secretion. Cell 121: 335-348, 2005.

11. Hasebe T, Sasaki S, Imoto S and Ochiai A: Highly proliferative fibroblasts forming fibrotic focus govern metastasis of invasive ductal carcinoma of the breast. Mod Pathol 14: 325-337, 2001.

12. Forsberg K, Valyi-Nagy I, Heldin CH, Herlyn M and Westermark B: Platelet-derived growth factor (PDGF) in oncogenesis: Development of a vascular connective tissue stroma in xenotransplanted human melanoma producing PDGF-BB. Proc Natl Acad Sci USA 90: 393-397, 1993.

13. Lindmark G, Sundberg C, Glimelius B, Påhlman L, Rubin K and Gerdin B: Stromal expression of platelet-derived growth factor beta-receptor and platelet-derived growth factor B-chain in colorectal cancer. Lab Invest 69: 682-689, 1993.

14. Shao ZM, Nguyen M and Barsky SH: Human breast carcinoma desmoplasia is PDGF initiated. Oncogene 19: 4337-4345, 2000.

15. Betsholtz C, Karlsson L and Lindahl P: Developmental roles of platelet-derived growth factors. Bioessays 23: 494-507, 2001.

16. Chen YQ, Ni DJ, Cheng Q, Hung HB, Meng Y and Wu MC: Study on the hypolipidemic effect of flavones and dihydromyricetin From Tengcha. J Tea Sci 3: 221-225, 242, 2007.

17. Zhong ZX, Zhou GF, Chen XF and Qin JP: Experimental study on the protective effect of dihydromyricetin from Guangxi Ampelopsis grossepentata on liver. Chin J Tradit Med Sci Technol 9: 155-156, 2002.

18. Xu JJ, Yao MJ and Wu MC: Study on biological efficacy of dihydromyricetin. Food Sci 29: 622-625, 2008

19. Shen Y, Lindemeyer AK, Gonzalez C, Shao XM, Spigelman I, Olsen RW and Liang J: Dihydromyricetin as a novel anti-alcohol intoxication medication. J Neurosci 32: 390-401, 2012.

20. He GX, Yang WL, Pei G, Zhu YH and Du FL: Studies on the effect of dihydromyricetin on antilipid-peroxidation. Zhongguo Zhong Yao Za Zhi 28: 1188-1190, 2003.

21. Mueller L, Goumas FA, Himpel S, Brilloff S, Rogiers X and Broering DC: Imatinib mesylate inhibits proliferation and modulates cytokine expression of human cancer-associated stromal fibroblasts from colorectal metastases. Cancer Lett 250: 329-338, 2007.

22. Gioni V, Karampinas T, Voutsinas G, Roussidis AE, Papadopoulos S, Karamanos NK and Kletsas D: Imatinib mesylate inhibits proliferation and exerts an antifibrotic effect in human breast stroma fibroblasts. Mol Cancer Res 6: 706-714, 2008.

23. Pietras K, Pahler J, Bergers G and Hanahan D: Functions of paracrine PDGF signaling in the proangiogenic tumor stroma revealed by pharmacological targeting. PLoS Med 5: e19, 2008.

24. Micke $\mathrm{P}$ and Ostman A: Tumour-stroma interaction: Cancerassociated fibroblasts as novel targets in anti-cancer therapy? Lung Cancer 45 (Suppl 2): S163-S175, 2004.

25. Kitadai Y, Sasaki T, Kuwai T, Nakamura T, Bucana CD, Hamilton SR and Fidler IJ: Expression of activated plateletderived growth factor receptor in stromal cells of human colon carcinomas is associated with metastatic potential. Int J Cancer 119: 2567-2574, 2006.

26. Tejada ML1, Yu L, Dong J, Jung K, Meng G, Peale FV, Frantz GD, Hall L, Liang X, Gerber HP and Ferrara N: Tumor-driven paracrine platelet-derived growth factor receptor alpha signaling is a key determinant of stromal cell recruitment in a model of human lung carcinoma. Clin Cancer Res 12: 2676-2688, 2006. 